\title{
Polyelectrolyte Multilayer Formation on Neutral Hydrophobic Surfaces
}

\author{
Juhyun Park ${ }^{\dagger}$ and Paula T. Hammond ${ }^{*}$
}

Department of Materials Science and Engineering and Department of Chemical Engineering, Massachusetts Institute of Technology, Cambridge, MA02139

\section{Fluorescence characteristics}

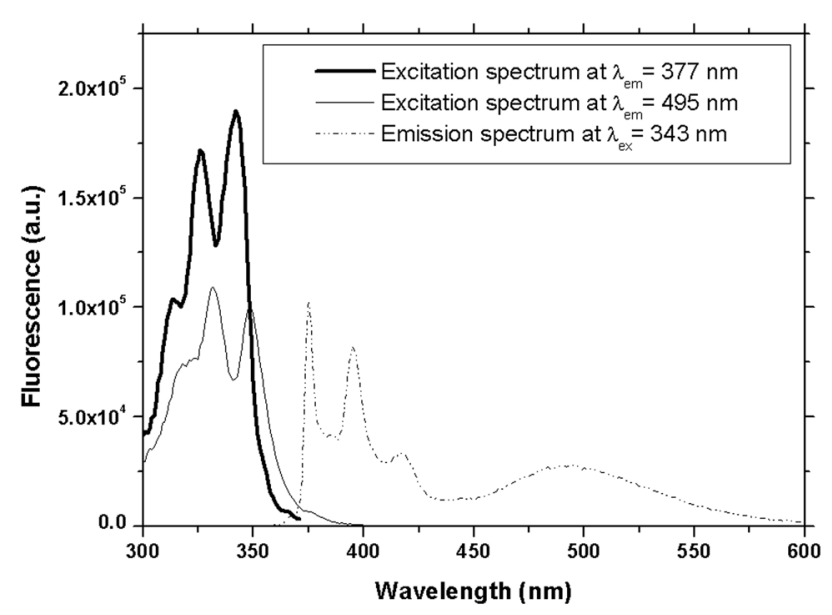

Figure S1. Excitation and emission spectra of $0.1 \mathrm{wt} \%$ PAH-Py2 solution at $\mathrm{pH} 6.14$ without added salts.

\section{Characterization of PAH-Py in Solutions}

2.1. Potentiometric Titration. $0.1 \mathrm{wt} \%$ of PAH and PAH-Py aqueous solutions (10.56 mM of PAH, $10.37 \mathrm{mM}$ of PAH-Py1, and $10.49 \mathrm{mM}$ of PAH-Py2) were prepared by dissolving PAH and PAH-Py into deionized water (initial $\mathrm{pH}=3.3 \sim 3.5$ ). A known amount of $\mathrm{HCl}$ was added to decrease solution $\mathrm{pH}$ down to 2 , and then titration of the polyelectrolyte solutions was performed with $0.1 \mathrm{M} \mathrm{NaOH}$ at $23^{\circ} \mathrm{C}$ with constant stirring, using a pH-meter purchased from Beckman Instruments, Inc. (Fullerton, CA). The volume of titrant subtracted by that of a blank titration under identical conditions was used for calculating the degree of ionization.

2.2. Degree of ionization. The hydrophobic polyelectrolyte, PAH used in this work is known to contain only primary amine groups, and thus is a weak polybase. The characteristic feature of the $\mathrm{PAH}-\mathrm{Py}$ ionization is that the degree of ionization decreases as the $\mathrm{pH}$ increases. The degree of ionization (protonation) was calculated from the electroneutrality condition, ${ }^{1,2}$

$$
\alpha(\%)=\frac{C_{H}^{\text {added }}-C_{O H}^{\text {added }}+C_{O H}^{\text {free }}-C_{H}^{\text {free }}}{C_{P}} \times 100
$$


where $C_{P}$ is the concentration of polyelectrolyte on a repeat unit basis; $C_{O H}^{\text {added }}$ is that of added $\mathrm{NaOH}$ calculated by subtracting the added amount for the blank titration; $C_{O H}^{\text {free }}$ and $C_{H}^{\text {free }}$ are those for the free protons and hydroxyl ions that were calculated from measured $\mathrm{pH}$ values neglecting corrections for the activity coefficient; $C_{H}^{\text {added }}$ refers to added $\mathrm{HCl}$ from the salt form of PAH-Py. Practically, the degree of salt formation could be less than $100 \%$ but we assumed that $C_{H}^{\text {added }}$ is equal to $C_{P}$ due to negligible imperfection $(\leq 2 \%)^{3}$ in the salt form. The values of $\mathrm{pKa}$ (we designated as $50 \%$ degree of ionization of the polyamine) measured by our potentiometric titration were 8.1 for both unlabeled PAH and PAH-Py. Both titration curves were coincident in the range shown in Figure 2, showing that less than 1 mol\% amine substitution by pyrene groups does not affect the original ionization behavior of unlabeled PAH; the introduced secondary amines due to the reductive amination and pyrene moieties did not change the degree of ionization of unlabeled PAH. The chains have varying degrees of ionization equal to $100 \%$

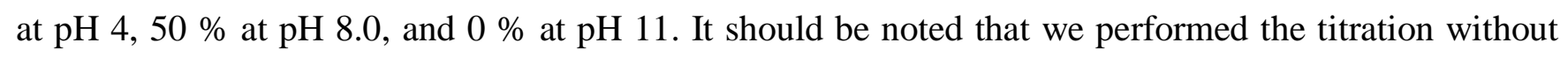
adding salt. Because salt might cause additional differences in the resulting titration curve due to varying ionic strength, we sought to follow the conditions that we use to control the degree of ionization of PAH chains by $\mathrm{pH}$ adjustment during adsorption. As a result, the titration curve is a bit broader than that with salt addition, but the pKa value is still between 8 and 9 which in a good agreement with literature values. ${ }^{3-7}$

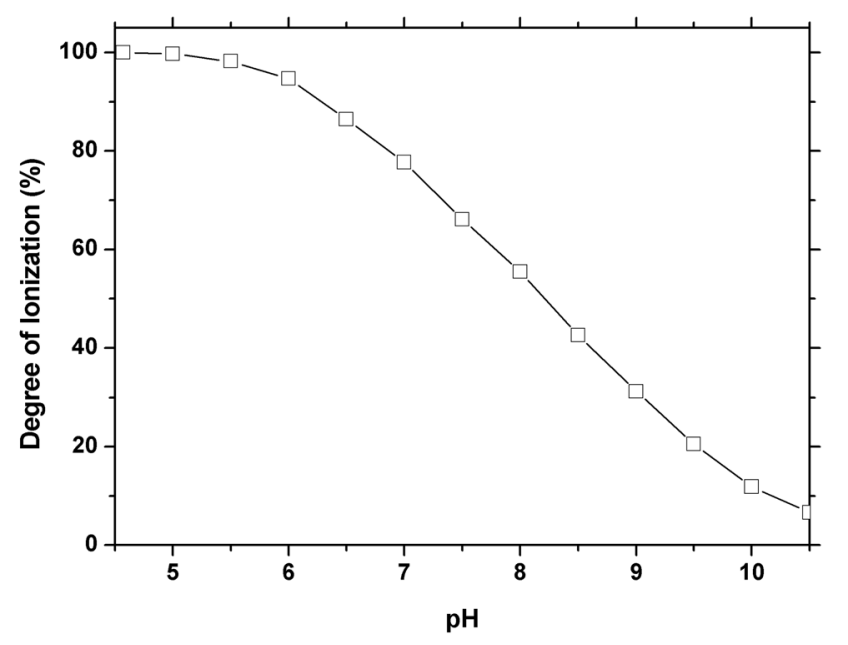

Figure S2. Potentiometric titration curve of $0.1 \mathrm{wt} \%$ PAH-Py1 aqueous solution.

\section{Adsorption theory of hydrophobic polyelectrolytes at neutral hydrophobic surfaces}

The theory can be applied in part to the specific case of this study by assuming negligible surface charge density. Before describing the adsorption behavior by giving an example of a semi-dilute adsorbed layer in the DR theory, it should be mentioned that hydroxyl ions can adsorb by penetration into hydrophobic surfaces, such as a self-assembled monolayer of alkyl chains or a solid surface of Teflon-AF, with enough lateral spaces or pores for their encapsulation, negatively charging the surfaces. ${ }^{8-10}$ However, we assume that this charging is not considerable compared to other predominant short-range interactions, although it would be critical in a strict consideration. When electrostatic attractions to the surface are negligible, the DR theory produces the following total free energy equation of the adsorbed layer, 


$$
\frac{F}{k T} \approx S l_{B} r_{D} f g_{\xi}\left(\frac{f g_{\xi}}{2 \xi^{4}} \exp \left(-\frac{\xi}{r_{D}}\right)\right)-S \varepsilon_{V W}{ }^{2} \frac{g_{\xi}}{\xi^{2}}
$$

where the free energy is $F$, the fraction of charged monomers is $f$, the Debye screening length is $r_{D}$, the Wigner-Seitz cell size is $\xi$, and the Bjerrum length is $l_{B}$, the sticking energy to the adsorbing surface is represented by $\varepsilon_{V W}$, the number of monomers inside the cell is $g_{\xi}$, and a surface area $S$ is the sum of the contribution from all chains. The first term in the right side of the equation represents the electrostatic repulsive contribution to the free energy, and the second term is the contribution from short-range attractive interactions.

An equation that shows the effect of two important variables, the ionic strength and the fraction of charged segments, on an adsorption property can be obtained by calculating the cell size $\xi$. When the free energy $F$ is minimized with respect to the cell size $\xi$, the equation that defines the cell size as a function of $r_{D}$ and $f$ is obtained as follows,

$$
\frac{f^{1 / 3}}{u^{1 / 3} \xi_{a}}\left(1+\frac{\xi}{2 r_{D}}\right) \exp \left(-\frac{\xi}{r_{D}}\right) \approx \frac{\varepsilon_{V W}^{2}}{l_{B} r_{D} f}
$$

where a bond size is $a$, and the ratio of the Bjerrum length to the bond size, $u$.

\section{Coiling index variation during multilayer assembly}

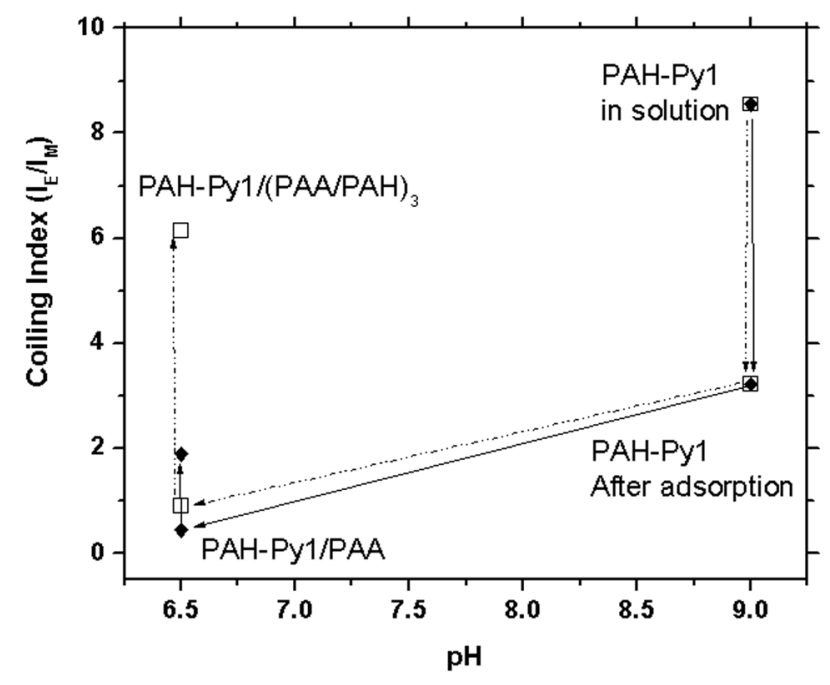

Figure S3. Coiling index variation of the PAH-Py1 prime layer during assembly process in the case of no added salt in the adsorption solutions (open square and dashed line) and when $0.1 \mathrm{M} \mathrm{NaCl}$ was added in the adsorption solutions (filled rhombus and solid line).

\section{Frequency shift of QCM during multilayer assembly}

SPS and PDAC were chosen to completely exclude the $\mathrm{pH}$ dependence in the degree of ionization of polyelectrolytes, in order to solely observe the effect of salt concentration in multilayering on a neutral hydrophobic surface. The frequency shift in QCM measurements is plotted as a function of the number of bilayers in Figure S4. Similar to multilayering SPS and PDAC on a charged surface, multilayer growth is the thickest at $0.1 \mathrm{NaCl}$ condition on our PAH-coated neutral hydrophobic surface. The 
importance of the PAH primary layer is also confirmed by building up multilayers comprised of SPS and PDAC without (open square in Figure S4) the PAH base layer. As SPS and PDAC have considerable hydrophobicity, they could be assembled on a neutral hydrophobic surface without the base layer when $0.1 \mathrm{M} \mathrm{NaCl}$ was added. However, the frequency shift was noticeably small compared to the case with the PAH base layer at the same ionic strength, proving that the hydrophobic weak polyelectrolyte, $\mathrm{PAH}$, was a better choice than a hydrophobic strong polyelectrolyte, PDAC, as a base layer for multilayering on the neutral hydrophobic surface.

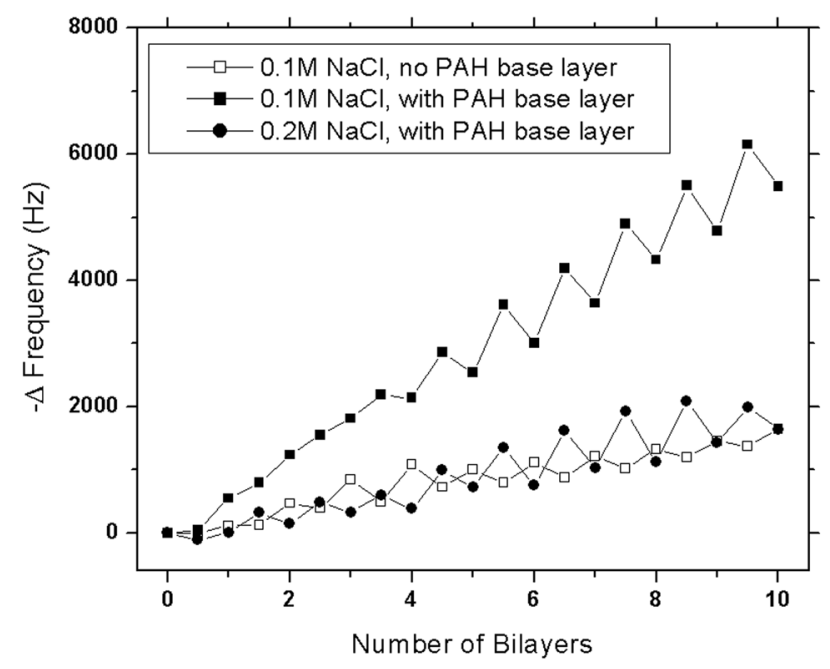

Figure S4. Frequency shift of QCM during a (SPS/PDAC) 10 multilayer assembly depending of the existence of the PAH base layer and salt concentrations in SPS and PDAC solutions.

\section{REFERENCES}

1. Mandel, M. Eur. Polym. J. 1970, 6, 807.

2. $\quad$ Smits, R. G.; Koper, G. J. M.; Mandel, M. J. Phys. Chem. 1993, 97, 5745.

3. Yoshikawa, Y.; Matsuoka, H.; Ise, N. Brit. Poly. J. 1986, 18, 242.

4. Choi, J.; Rubner, M. F. Macromolecules 2005, 38, 116.

5. Petrov, A. I.; Antipov, A. A.; Sukhorukov, G. B. Macromolecules 2003, 36, 10079.

6. Ochiai, H.; Anabuki, Y.; Kojima, O.; Tominaga, K.; Murakami, I. J. Poly. Sci. Part B-Poly. Phys. 1990, 28, 233.

7. Fang, M. M.; Kim, C. H.; Saupe, G. B.; Kim, H. N.; Waraksa, C. C.; Miwa, T.; Fujishima, A.; Mallouk, T. E. Chem. Mater. 1999, 11, 1526.

8. $\quad$ Kreuzer, H. J.; Wang, R. L. C.; Grunze, M. J. Am. Chem. Soc. 2003, 125, 8384.

9. Quinn, A.; Sedev, R.; Ralston, J. J. Phys. Chem. B 2003, 107, 1163.

10. Zimmermann, R.; Dukhin, S.; Werner, C. J. Phys. Chem. B 2001, 105, 8544. 